\title{
Prevalence of Elevated Alanine Aminotransferase by Diagnostic Criterion, Age, and Gender among Adolescents
}

\author{
Jing Zhang, ${ }^{1}$ Zheng-Ying Wang, ${ }^{1}$ Jing-Ping Zhang, ${ }^{2}$ Hua Zhou, ${ }^{3}$ and Zan Ding $\mathbb{D}^{3}$ \\ ${ }^{1}$ Department of Nursing, Baoan Central Hospital of Shenzhen, The Fifth Affiliated Hospital of Shenzhen University, Shenzhen, \\ Guangdong 518102, China \\ ${ }^{2}$ The Institute of Nursing Psychological Research Center, Xiangya College of Nursing, Central South University, Changsha, \\ Hunan 410013, China \\ ${ }^{3}$ Institute of Low Carb Medicine, Baoan Central Hospital of Shenzhen, The Fifth Affiliated Hospital of Shenzhen University, \\ Shenzhen, Guangdong 518102, China
}

Correspondence should be addressed to Zan Ding; dingzan_1990@163.com

Received 16 August 2019; Accepted 28 December 2019; Published 25 January 2020

Academic Editor: Vincenzo Pilone

Copyright (c) 2020 Jing Zhang et al. This is an open access article distributed under the Creative Commons Attribution License, which permits unrestricted use, distribution, and reproduction in any medium, provided the original work is properly cited.

\begin{abstract}
Background. Serum alanine aminotransferase (ALT) activity was measured not only to detect liver disease, but also to monitor overall health. The purpose of this study was to obtain the prevalence of elevated ALT levels among adolescents. Methods. In a school-based cross-sectional study, a representative sample was analyzed from 9 middle and high schools in Shenzhen, China, during 2017 to 2018. Elevated ALT was defined as diagnostic criterion I ( $>30 \mathrm{U} / \mathrm{L}$ for boys and >19 U/L for girls) and diagnostic criterion II (>40 U/L). Results. From the adolescent population, a total of 7281 students (boys, 4014, and girls, 3267) aged from 10 to 17 years were collected. The prevalence of elevated ALT was $7.11 \%$ (6.88\% for boys and $7.41 \%$ for girls) by criterion I and $2.72 \%$ (3.96\% for boys and 1.19\% for girls) by criterion II. Based on the Shenzhen census and Chinese national census population, the adjusted prevalence of elevated ALT was 7.65\% (boys $7.19 \%$ and girls $8.21 \%$ ) and $6.79 \%$ (boys $6.07 \%$ and girls $7.56 \%$ ) by criterion I and $2.85 \%$ (boys $4.20 \%$ and girls $1.16 \%$ ) and $2.43 \%$ (boys $3.49 \%$ and girls $1.29 \%$ ) by criterion II. For age, the overall trends were increasing progressively, regardless of the use of diagnostic criteria for an elevated ALT activity. Conclusions. This study supplements the gap that the prevalence of elevated ALT levels differed in gender, age, and criteria among adolescents of Shenzhen. We should take the prevalence as a predictor and continue to play a warning and preventive role in preparation for further intervention.
\end{abstract}

\section{Introduction}

Serum alanine aminotransferase (ALT) is a liver enzyme in the cytosol of hepatocytes, and the elevated values are frequently used to evaluate liver dysfunction [1]. As an indicator of health and disease [2], ALT has been proven several times to be a sensitive biochemical marker to screen for the detection of possible diseases, such as cardiovascular complications [3], osteopenia, bone mineral density [4], obstructive sleep apnea-hypopnea syndrome [5], anorexia nervosa [6], and depression [7]. As a part of annual check-ups, ALT levels increased after different kinds of liver injuries and when at risk of hepatitis, liver cirrhosis, liver steatosis, nonalcoholic steatohepatitis, hepatic hypoperfusion, and nonalcoholic fatty liver disease (NAFLD), which were more common in individuals with elevated ALT than with normal ALT $[8,9]$. There were different causes of elevated ALT, including obesity-related diseases, hypertension, hyperglycemia, hypertriglyceridemia, diabetes, medication use, and metabolic syndrome $[10,11]$. It is necessary to evaluate the prevalence of elevated ALT, which could be used as one of the surrogate markers of overall health in a general population.

Numerous national studies found that the prevalence of elevated ALT levels among adults and adolescents varied from different countries, ethnic groups, gender, age, and different diagnostic criteria [12-16]. Among adolescents, the prevalence of elevated ALT was shown in the U.S. [17-20], Korea [12, 21], Mexico [22, 23], China [10], Netherlands 
[24], and Iran [25]. The considerably different prevalence had pointed out the importance of standardizing and documenting the variations of ALT levels. Moreover, adolescents have been one of the group of greatest concern to society, because due to rapid growth at youth, the abnormalALT elevation may have further influence among adolescents than adults in the future for overall health $[1,26]$.

However, the prevalence of elevated ALT among adolescents had not been adequately evaluated in mainland China, especially in the new metropolis of Shenzhen. As one of the super bigger and newest first-tier cities worldwide, the total area of Shenzhen was $1997.3 \mathrm{~km}^{2}$ and the population was around 11.37 million with about $70 \%$ being migrants. Therefore, we really need to implement a clinical survey and to analyze the prevalence of elevated ALT among the adolescents of Shenzhen, China, in a cross-sectional study. The objectives of the present study were to examine the crude and standardized prevalence of elevated ALT for the total adolescent group as well as the subgroups by age and gender and to summarize the prevalence of elevated ALT among adolescents all over the world.

\section{Materials and Methods}

2.1. Study Population. The current study was conducted in Shenzhen, located in the Pearl River Delta region in southern China. As the first special economic zone of China and one of the largest manufacturing bases in the world, Shenzhen has a total gross domestic product (GDP) of 0.27 trillion USD and 24,640 USD per capita in 2015.

We implemented a school-based descriptive survey and recruited all the freshmen from 9 junior and senior high schools (HangCheng School, Baoan Vocational Technical School, Oriental English School, HeZhou School, Huang$\mathrm{MaBu}$ School, JinBi Experimental School, KangQiao School, LongShan School, and ZhongAo Experimental School) [27]. The whole clinical data were extracted from the compulsory admission physical examination organized by the Baoan District Government of Shenzhen from February 2017 to June 2018. In this study, adolescents were defined as $10-17$ years old. A total of 7382 students initially participated in our study; 28 cases $\geq 18$ years old, 16 cases $<10$ years old, 41 cases lacked ALT, and 16 cases with missed age were deleted. Finally, 7281 valid samples remained (effective rate $98.6 \%$ ).

2.2. Data Collection. Demographic information such as age, sex, and grade were obtained from self-administered questionnaires. Anthropometric and laboratory measurements of students were also taken. Height and weight were measured by trained physicians to the nearest $0.5 \mathrm{~cm}$ and $0.1 \mathrm{~kg}$, without shoes, socks, and any other heavy clothing. After an overnight fast of $10 \mathrm{~h}$, blood samples were drawn from an antecubital vein in each subject into vacutainer tubes. The tubes were delivered to the laboratory of the hospital in an insulated box with ice by a specially assigned physician and centrifuged within an hour. ALT was measured using the AU5800 serial with the automatic biochemical analyzer
(Model AU5821; Tokyo, Japan) by the manufacturing enterprise Beckman Coulter K.K. Written informed consents for each participant and their parents were obtained.

2.3. Definition of Elevated Serum ALT Levels. For adolescents, the common definitions of elevated ALT used were above $30 \mathrm{U} / \mathrm{L}$ for boys and above 19U/L for girls [25, 28]. Besides, ALT values were quantitatively estimated above $40 \mathrm{U} / \mathrm{L}$ in previous studies $[19,22]$. In the present study, the prevalence of elevated ALT was mainly diagnosed by ALT $>30 \mathrm{U} / \mathrm{L}$ for boys and $>19 \mathrm{U} / \mathrm{L}$ for girls (i.e., criterion I) and ALT $>40 \mathrm{U} / \mathrm{L}$ for both sexes (i.e., criterion II).

2.4. Statistical Analysis. The normal distribution of serum ALT was determined by the Shapiro-Wilk test. Significance for intergroup differences was evaluated by the MannWhitney $U$ test for the skewed blood index. We combined 10-11-year-old adolescents in the statistical analysis of the ALT abnormality rate, because the sample size of the 10 year olds was too small. The prevalence of elevated ALT with 95\% confidence interval (95\% CI) was quantitatively estimated, diagnosed by both criteria I and II. Significance for intergroup difference between boys and girls in the prevalence of elevated ALT was assessed by Pearson's chi-square test. A chi-square test for the trend of the crude age-prevalence of elevated ALT was also performed. Age- and/or sexstandardized prevalence rates of elevated ALT were estimated by the direct standardization method, according to the distribution of the population in the 2010 Shenzhen population census (http://www.sztj.gov.cn/xxgk/zfxxgkml/tjsj) as well as the 2010 Chinese population census (http://www .stats.gov.cn/tjsj). Associations with age and gender were examined using multiple logistic regression models, expressed as odds ratios and 95\% CIs for risk of elevated ALT.

All statistical analyses involved use of SPSS for Windows 13.0 (SPSS Inc., Chicago, IL, USA) and R software version 3.2.0 (http://www.R-project.org, the R Foundation for Statistical Computing, Vienna, Austria), and graphics were carried out by SigmaPlot software version 10.0. Two-tailed tests of significance were reported, and $P<0.05$ was considered statistically significant.

\section{Results}

3.1. Sample Characteristics. Overall, we collected 7281 adolescents aged 10-17 years for analysis, involving 4014 boys and 3267 girls. Table 1 shows the baseline descriptive statistics for the levels of ALT stratified by age and gender. For 10-17 year olds, the mean (standard deviation) value of ALT was separately 15.96 (17.02), 12.18 (11.06), and 14.26 (14.77) U/L for boys, girls, and both genders, respectively. Boys had higher ALT levels than girls $(P<0.001)$. The concentrations of serum ALT were not normally distributed $(P<0.001)$. The 5th, 25th, 75th, and 95th percentiles of serum ALT levels were 7, 9, 15, and $30 \mathrm{U} / \mathrm{L}$ for overall participants; 7, 10, 16, and $37 \mathrm{U} / \mathrm{L}$ for boys; and 6, 9, 13, and $22 \mathrm{U} / \mathrm{L}$ for girls, respectively. 
TABLE 1: Baseline descriptive statistics for serum alanine aminotransferase levels (U/L) stratified by gender among the adolescents (aged 1017 years) of Shenzhen, China, 2017-2018.

\begin{tabular}{|c|c|c|c|c|c|c|c|c|c|c|c|c|c|}
\hline \multirow{2}{*}{ Sample } & \multirow{2}{*}{$N$} & \multirow{2}{*}{ Mean } & \multirow{2}{*}{$\mathrm{SD}$} & \multirow{2}{*}{ Min } & \multicolumn{8}{|c|}{ Percentiles } & \multirow{2}{*}{ Max } \\
\hline & & & & & 5 th & 10th & 25 th & 50 th & 75th & 90th & 95th & 99th & \\
\hline \multicolumn{14}{|c|}{ Overall (years) } \\
\hline $10.0-10.9$ & 60 & 11.25 & 5.04 & 3 & 6 & 7 & 9 & 10 & 13 & 14 & 15 & 33 & 39 \\
\hline $11.0-11.9$ & 967 & 12.23 & 6.85 & 3 & 7 & 8 & 9 & 11 & 14 & 17 & 20 & 38 & 141 \\
\hline $12.0-12.9$ & 1511 & 12.67 & 9.29 & 1 & 7 & 8 & 9 & 11 & 13 & 18 & 22 & 48 & 206 \\
\hline $13.0-13.9$ & 471 & 14.85 & 21.84 & 3 & 7 & 8 & 9 & 12 & 15 & 20 & 27 & 76 & 415 \\
\hline $14.0-14.9$ & 1101 & 14.21 & 12.76 & 1 & 7 & 8 & 9 & 11 & 14 & 22 & 33 & 68 & 193 \\
\hline $15.0-15.9$ & 1789 & 15.57 & 19.59 & 3 & 7 & 8 & 9 & 11 & 16 & 24 & 35 & 75 & 495 \\
\hline $16.0-16.9$ & 1136 & 15.80 & 15.54 & 3 & 7 & 7 & 9 & 12 & 16 & 25 & 36 & 81 & 213 \\
\hline $17.0-17.9$ & 246 & 15.29 & 11.38 & 4 & 7 & 8 & 9 & 12 & 16 & 25 & 33 & 65 & 93 \\
\hline $10.0-17.9$ & 7281 & 14.26 & 14.77 & 1 & 7 & 8 & 9 & 11 & 15 & 21 & 30 & 67 & 495 \\
\hline \multicolumn{14}{|l|}{ Boys (years) } \\
\hline $10.0-10.9$ & 33 & 11.21 & 5.58 & 6 & 6 & 7 & 9 & 10 & 12 & 14 & 15 & 31 & 39 \\
\hline $11.0-11.9$ & 490 & 13.36 & 8.52 & 4 & 7 & 8 & 10 & 12 & 15 & 18 & 23 & 46 & 141 \\
\hline $12.0-12.9$ & 899 & 13.39 & 8.80 & 2 & 7 & 8 & 9 & 11 & 14 & 19 & 25 & 50 & 113 \\
\hline $13.0-13.9$ & 269 & 13.17 & 10.12 & 4 & 7 & 8 & 9 & 11 & 14 & 19 & 25 & 50 & 138 \\
\hline $14.0-14.9$ & 546 & 16.74 & 16.36 & 1 & 7 & 8 & 10 & 12 & 18 & 27 & 40 & 92 & 193 \\
\hline $15.0-15.9$ & 1017 & 18.23 & 24.31 & 3 & 7 & 8 & 10 & 13 & 18 & 29 & 40 & 116 & 495 \\
\hline $16.0-16.9$ & 624 & 18.51 & 19.45 & 3 & 7 & 8 & 10 & 13 & 19 & 33 & 50 & 102 & 213 \\
\hline $17.0-17.9$ & 136 & 17.16 & 13.62 & 4 & 7 & 8 & 10 & 12 & 18 & 32 & 49 & 65 & 93 \\
\hline $10.0-17.9$ & 4014 & 15.96 & 17.02 & 1 & 7 & 8 & 10 & 12 & 16 & 25 & 37 & 79 & 495 \\
\hline \multicolumn{14}{|l|}{ Girls (years) } \\
\hline $10.0-10.9$ & 27 & 11.30 & 4.39 & 3 & 7 & 7 & 10 & 11 & 13 & 14 & 16 & 25 & 28 \\
\hline $11.0-11.9$ & 477 & 11.07 & 4.24 & 3 & 6 & 7 & 9 & 10 & 12 & 16 & 18 & 27 & 40 \\
\hline $12.0-12.9$ & 612 & 11.62 & 9.89 & 1 & 6 & 7 & 9 & 10 & 12 & 16 & 19 & 43 & 206 \\
\hline $13.0-13.9$ & 202 & 17.07 & 31.15 & 3 & 7 & 8 & 9 & 12 & 16 & 22 & 30 & 96 & 415 \\
\hline $14.0-14.9$ & 555 & 11.72 & 6.89 & 2 & 6 & 7 & 8 & 10 & 13 & 16 & 21 & 43 & 68 \\
\hline $15.0-15.9$ & 772 & 12.06 & 9.48 & 3 & 6 & 7 & 8 & 10 & 13 & 18 & 23 & 46 & 186 \\
\hline $16.0-16.9$ & 512 & 12.51 & 7.44 & 3 & 7 & 7 & 9 & 11 & 14 & 20 & 24 & 36 & 88 \\
\hline $17.0-17.9$ & 110 & 12.98 & 7.17 & 4 & 7 & 8 & 9 & 12 & 15 & 19 & 22 & 29 & 69 \\
\hline $10.0-17.9$ & 3267 & 12.18 & 11.06 & 1 & 6 & 7 & 9 & 10 & 13 & 17 & 22 & 46 & 415 \\
\hline
\end{tabular}

ALT: alanine aminotransferase; SD: standard deviation.

3.2. Crude Prevalence of Elevated ALT. As shown in Table S1, a total of 518 and 198 adolescents had abnormal ALT levels separately, based on the diagnostic criteria I ( $>30 \mathrm{U} / \mathrm{L}$ for boys and $>19 \mathrm{U} / \mathrm{L}$ for girls) and II $(>40 \mathrm{U} / \mathrm{L})$. The crude age-prevalence of elevated ALT among adolescents was $7.11 \%$ (6.88\% for boys and $7.41 \%$ for girls) by criterion I and $2.72 \%$ (3.96\% for boys and $1.19 \%$ for girls) by criterion II. Obviously, the prevalence of elevated ALT, influenced by gender and age, was higher by criterion I than by criterion II. In addition, Figure 1 shows the trends of the crude ageprevalence of elevated ALT, which displayed an uptrend by age (both $P<0.001$ ).

3.3. Standardized Prevalence of Elevated ALT. According to the Shenzhen census, the overall standardized prevalence of elevated ALT was $7.65 \%$ (boys $7.19 \%$ and girls $8.21 \%$ ) by criterion I and $2.85 \%$ (boys $4.20 \%$ and girls $1.16 \%$ ) by criterion
II; based on the Chinese national census, the standardized prevalence was $6.79 \%$ (boys $6.07 \%$ and girls $7.56 \%$ ) by criterion I and $2.43 \%$ (boys $3.49 \%$ and girls $1.29 \%$ ) by criterion II (Figure 2). The standardized prevalence of elevated ALT was similar with the results of crude prevalence shown in Table 2 .

3.4. Univariable and Multivariable Logistic Regression Analyses. Tables 3 and 4 show the outcomes of gender and age difference with elevated ALT by logistic regression analysis. For gender, the abnormal rate of ALT in girls was much higher than that in boys $(\mathrm{OR}=1.65,95 \% \mathrm{CI}$ : $1.35-2.03)$ by criterion I, but lower in girls $(0.42,0.29-0.60)$ by criterion II, after being adjusted for age and body mass index using a multivariable logistic regression. For age, compared to the reference group, the overall trends were increasing progressively, regardless of the use of diagnostic criteria for an elevated ALT activity. 


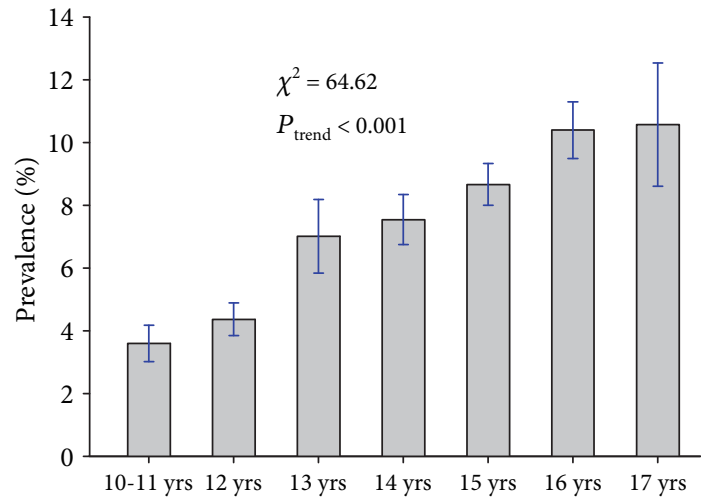

(a)

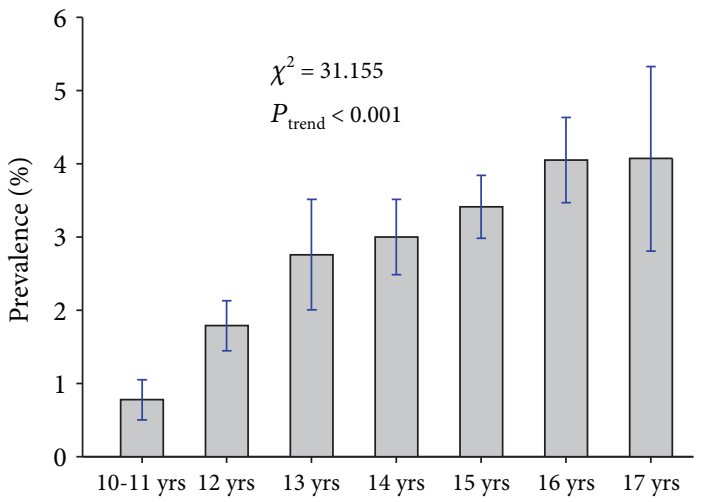

(b)

FIgURE 1: Crude age-prevalence of elevated alanine aminotransferase (ALT) based on the diagnostic criteria I (a) and II (b) among the adolescents of Shenzhen, China, 2017-2018.

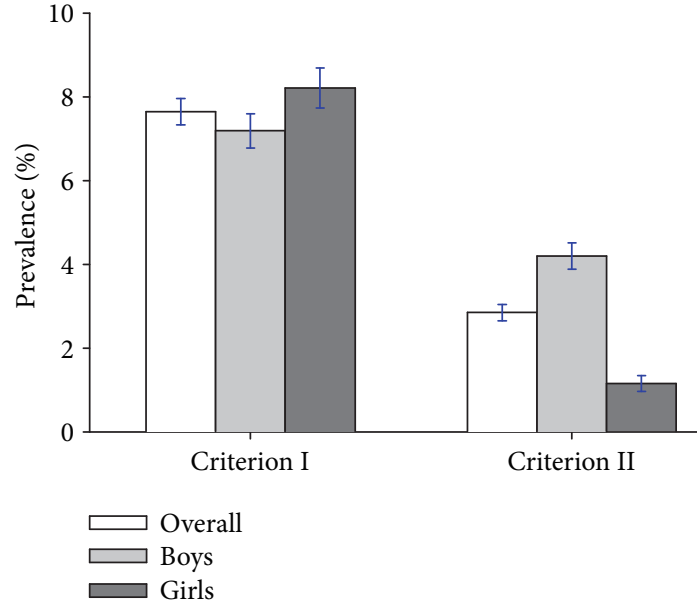

(a)

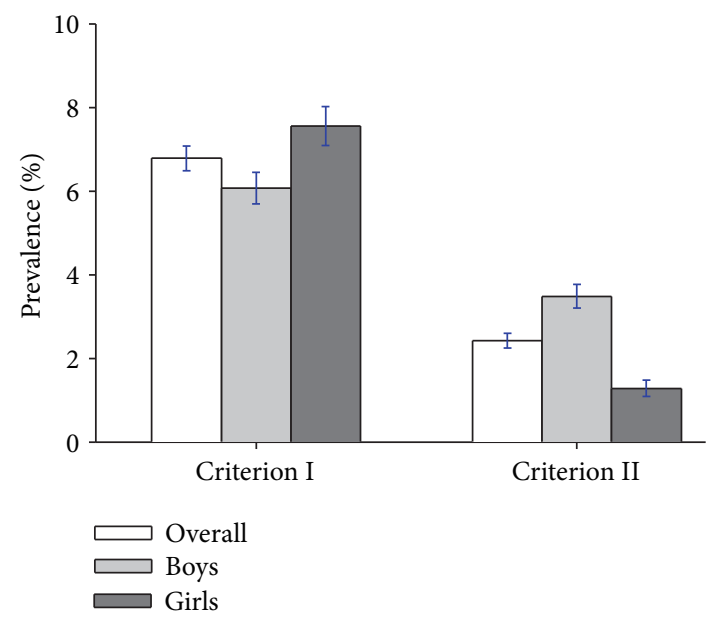

(b)

FIGURE 2: Standardized prevalence of elevated alanine aminotransferase (ALT) among the adolescents of Shenzhen, China, 2017-2018, diagnosed with both the criteria I and II, based on the 2010 Shenzhen Census Population (a) and the 2010 Chinese National Census Population (b).

3.5. The Prevalence of Elevated ALT among Adolescents Worldwide. In Table 5, we summarized the prevalence of elevated ALT among adolescents worldwide. The prevalence of elevated ALT among adolescents differed by the sex ratio and age of objects, area, ethnicity, study period, and diagnostic criterion $[19,22,23,29-31]$.

\section{Discussion}

Shenzhen is a vibrant city with a large number of immigrants, and the majority of the population here lives in a fast-paced and rapid-developing social environment [32]. This study examined the crude and standardized prevalence of elevated ALT among adolescents of Shenzhen for the total group and subgroups, showing that the prevalence of elevated ALT increased with age and differed by gender at puberty. The levels of ALT may be influenced by different variations including region, ethnic groups, overweight, obesity, liver diseases, and other factors, and we therefore summarized the prevalence of elevated ALT among adolescents on a global scale.

4.1. Diagnostic Criteria for Elevated ALT Levels. Nowadays, almost all countries use gender-specific criteria to determine the elevated ALT levels rather than the single standard usually used $[18,21,33]$. Our results obtained the prevalence of elevated ALT for boys and girls in diagnostic criteria I (>30 U/L for boys and >19 U/L for girls) and II (>40 U/L). Aside from the two main criteria above used in our study, there were other criteria (e.g., ALT $>33 \mathrm{U} / \mathrm{L}$ for boys and $>25 \mathrm{U} / \mathrm{L}$ for girls [33], $>30 \mathrm{U} / \mathrm{L}$ for boys and $>21 \mathrm{U} / \mathrm{L}$ for girls [34], and >30 U/L for both sexes [24]) with which to diagnose an elevated ALT activity.

4.2. The Prevalence of Elevated ALT. Based on various criteria, the abnormal rates of elevated ALT obviously differed. Among the adolescents of Shenzhen, the prevalence of elevated ALT was $7.11 \%$ by criterion I and $2.72 \%$ by criterion II; in Korea, $7.9 \%$ for boys and $10.2 \%$ for girls by criterion I 


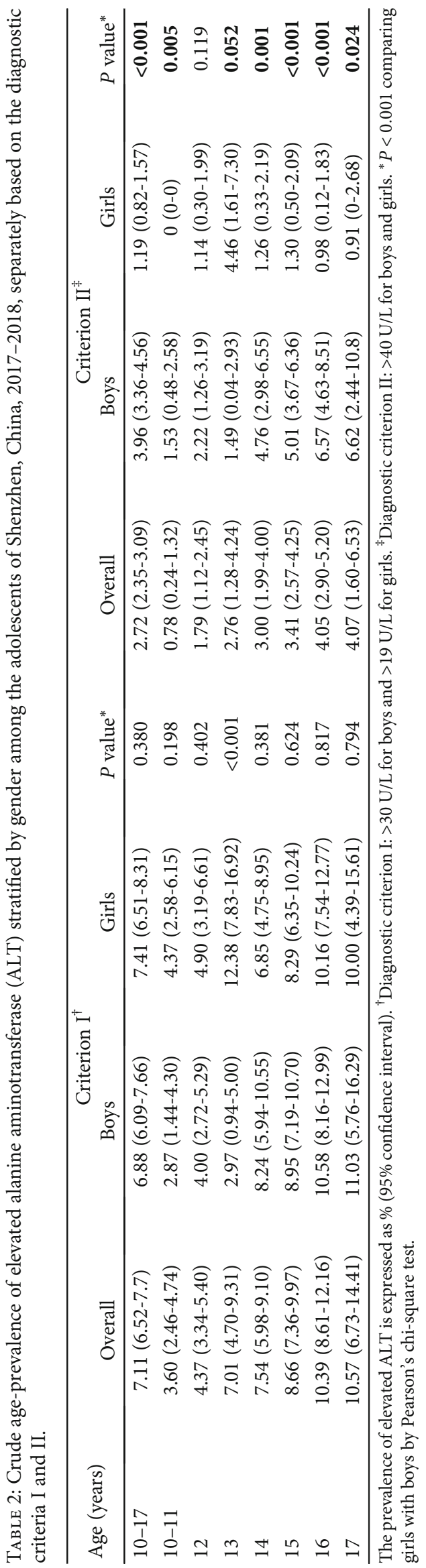


TABLE 3: Univariate and multivariate logistic regression analyses with elevated alanine aminotransferase (ALT) as the outcome variable, based on the diagnostic criterion I ( $>30 \mathrm{U} / \mathrm{L}$ for boys and $>19 \mathrm{U} / \mathrm{L}$ for girls).

\begin{tabular}{|c|c|c|c|c|c|c|}
\hline \multirow{2}{*}{ Variable } & \multicolumn{3}{|c|}{ Univariate analysis } & \multicolumn{3}{|c|}{ Multivariate analysis $^{\mathrm{a}}$} \\
\hline & Wald test & OR $(95 \%$ CI $)$ & $P$ & Wald test & OR (95\% CI) & $P$ \\
\hline Girls (vs. boys) & 0.770 & $1.08(0.91-1.30)$ & 0.380 & 23.587 & $1.65(1.35-2.03)$ & $<0.001$ \\
\hline Age (years) & 62.625 & & $<0.001$ & 34.547 & & $<0.001$ \\
\hline $10-11$ & & 1.00 & & & 1.00 & \\
\hline 12 & 0.917 & $1.22(0.81-1.84)$ & 0.338 & 1.295 & $1.29(0.83-2.01)$ & 0.255 \\
\hline 13 & 8.108 & $2.02(1.24-3.27)$ & 0.004 & 7.431 & $2.06(1.23-3.47)$ & 0.006 \\
\hline 14 & 14.816 & $2.18(1.47-3.25)$ & $<0.001$ & 5.601 & $1.68(1.09-2.59)$ & 0.018 \\
\hline 15 & 24.716 & $2.54(1.76-3.66)$ & $<0.001$ & 12.089 & $2.03(1.36-3.02)$ & 0.001 \\
\hline 16 & 34.169 & $3.10(2.12-4.53)$ & $<0.001$ & 19.535 & $2.53(1.67-3.83)$ & $<0.001$ \\
\hline 17 & 18.656 & $3.16(1.88-5.33)$ & $<0.001$ & 15.973 & $3.17(1.80-5.58)$ & $<0.001$ \\
\hline Constant & - & - & - & 667.963 & - & $<0.001$ \\
\hline
\end{tabular}

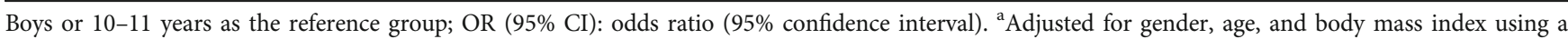
multivariable logistic regression analysis.

TABLE 4: Univariate and multivariate logistic regression analyses with elevated alanine aminotransferase (ALT) as the outcome variable, based on the diagnostic criterion II ( $>40 \mathrm{U} / \mathrm{L}$ for boys and girls).

\begin{tabular}{|c|c|c|c|c|c|c|}
\hline \multirow{2}{*}{ Variable } & \multicolumn{3}{|c|}{ Univariate analysis } & \multicolumn{3}{|c|}{ Multivariate analysis $^{\mathrm{a}}$} \\
\hline & Wald test & OR $(95 \%$ CI $)$ & $P$ value & Wald test & OR (95\% CI) & $P$ value \\
\hline Girls (vs. boys) & 46.388 & $0.29(0.21-0.42)$ & $<0.001$ & 21.129 & $0.42(0.29-0.60)$ & $<0.001$ \\
\hline Age (years) & 28.711 & & $<0.001$ & 16.540 & & 0.011 \\
\hline $10-11$ & & 1.00 & & & 1.00 & \\
\hline 12 & 4.315 & $2.32(1.05-5.12)$ & 0.038 & 3.872 & $2.32(1.00-5.38)$ & 0.049 \\
\hline 13 & 8.054 & $3.62(1.49-8.78)$ & 0.005 & 9.492 & $4.31(1.70-10.92)$ & 0.002 \\
\hline 14 & 11.940 & $3.94(1.81-8.56)$ & 0.001 & 7.945 & $3.24(1.43-7.35)$ & 0.005 \\
\hline 15 & 15.808 & $4.50(2.14-9.43)$ & $<0.001$ & 8.593 & $3.21(1.47-6.99)$ & 0.003 \\
\hline 16 & 19.030 & $5.38(2.53-11.44)$ & $<0.001$ & 11.086 & $3.90(1.75-8.69)$ & 0.001 \\
\hline 17 & 12.346 & $5.40(2.11-13.82)$ & $<0.001$ & 10.645 & $5.34(1.95-14.60)$ & 0.001 \\
\hline Constant & - & - & - & 268.333 & - & $<0.001$ \\
\hline
\end{tabular}

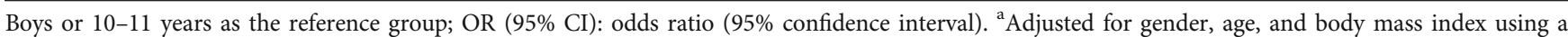
multivariable logistic regression analysis.

[28] and 3.6\% for boys and $2.8 \%$ for girls by criterion II [12]; and in Hong Kong of China, 3.2\% by ALT $>40 \mathrm{U} / \mathrm{L}$ and $5.9 \%$ by $\mathrm{ALT}>30 \mathrm{U} / \mathrm{L}$ [35].

From Table 5, the prevalence of elevated ALT among adolescents ranged from $1.5 \%$ in mainland China for girls [10] to $34.9 \%$ in Korea for obese populations [33]. The prevalence of elevated ALT is diverse in different national groups, such as $4.9 \%$ for boys and $7.5 \%$ for girls in Iran by criterion I [25], 3.0\% in Mexico (>40 U/L) [22], $8.0 \%$ in the U.S. $(>30 \mathrm{U} / \mathrm{L})$ [19], $3.1 \%$ in China $(\geq 40 \mathrm{U} / \mathrm{L})$ [10], and $5.3 \%$ $(>30 \mathrm{U} / \mathrm{L})$ and $2.8 \%(>40 \mathrm{U} / \mathrm{L})$ in Korea [34].

4.3. Prevalence of Elevated ALT Differed by Gender. Gender differences turned up in our study; for example, the proportion of the abnormal rate was higher for girls than boys by criterion I ( $7.41 \%$ vs. $6.88 \%$, respectively) but lower by criterion II (1.19\% vs. $3.96 \%$, respectively). Evaluating the abnormal ALT levels by gender shows more individuality than gender-neutral assessment. Most studies reported different prevalence rates of elevated ALT by gender (e.g., $8.0 \%$ for boys and 2.1\% for girls in Korea [21], 5.6\% for boys and $1.6 \%$ for girls in the U.S. [19], $4.8 \%$ for boys and $1.5 \%$ for girls in mainland China [10], and 9.8\% for boys and 3.8\% for girls in Mexico [23]).

The levels of serum ALT were higher in boys than in girls, which may relate to the differences in muscle mass and endogenous hormone levels (self-undetermined physical and emotional factors) between genders [36-39]. In this regard, it is well acknowledged that serum concentrations of estradiol are low in preadolescent girls and increase at menarche $[40,41]$. The age-related hormonal changes could partly account for the ALT levels that we observed between genders.

4.4. Prevalence of Elevated ALT Differed by Age. With the metabolic liver diseases gradually becoming younger currently, the abnormal rates of elevated ALT clearly reflected an uptrend with growing age. Focused on the Shenzhen adolescents of 10-17 years, the prevalence of elevated ALT was a rising tendency with increasing age, regardless of the use of 


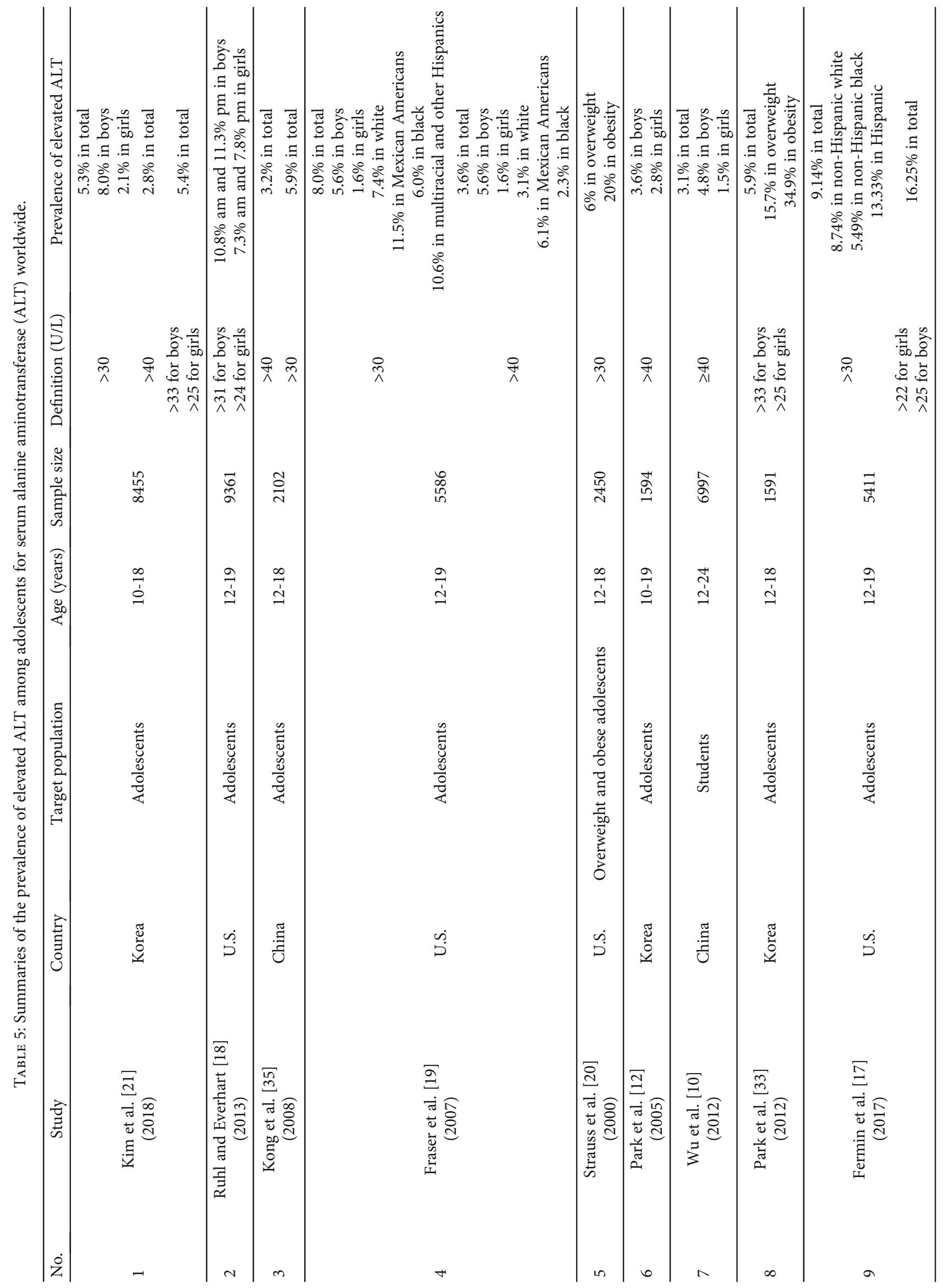




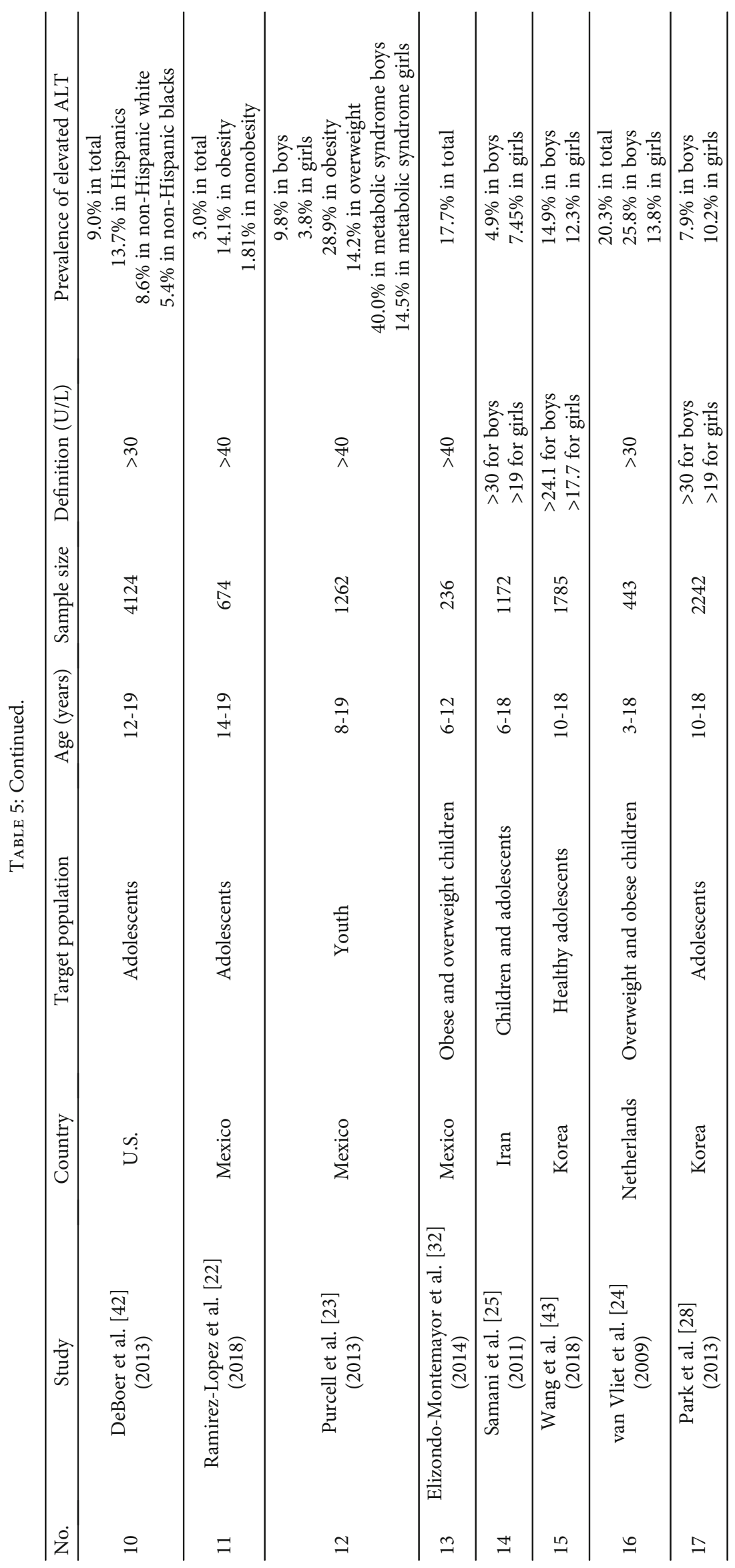


criterion I or II. For adults, the Rancho Bernardo Study cohort showed that the levels of ALT were highest in a young group (30-62 years) and subsequently decreased with increasing age among 63-93 years [16]; another longitudinal study also reported similar findings that the mean levels of ALT decreased with age in elders, with a mean age of 65.7 years [29]. From the above two studies, we could know the downtrend with age of ALT levels in the elderly age range. Containing 335 subjects ( $\geq 18$ years), a Jerusalem study on age-ALT association reported the peaking age (40-55 years) from an inverted $U$ curve [15]. Thus, uptrend with age in the adolescent period should probably be taken into account.

4.5. Other Influencing Factors for Abnormal ALT Levels. Except for the above four factors (i.e., diagnostic criterion, age, gender, and region), ethnicity may be a potential influencing factor to affect the prevalence of elevated ALT levels. It was significant to note that ethnics affect the abnormal rate in several studies (e.g., 13.7\% in Hispanics, $8.6 \%$ in non-Hispanic whites, and $5.4 \%$ in non-Hispanic blacks [42]; $7.4 \%$ among whites, $6.0 \%$ among blacks, $11.5 \%$ in Mexican Americans, and $10.6 \%$ in multiracial and other Hispanic adolescents [19]).

It has been reported that ALT levels and the prevalence of elevated ALT were more common in overweight or obese adolescents (e.g., $6 \%$ of the overweight and $20 \%$ of the obese in the U.S. [20], $15.7 \%$ of the overweight and $34.9 \%$ of the obese in Korea [16], and $14.2 \%$ of the overweight and 28.9\% of the obese in Mexico [33]).

The levels of ALT were closely connected with the damage of hepatocyte and hepatic enzymes. Elevated ALT played a predictive role in the diagnosis of liver diseases (e.g., NAFLD and metabolic liver diseases), which were prevalent but concealed in adolescents [43]. Furthermore, bad living habits (e.g., staying up late, lack of exercise, and increasingly sedentary lifestyle), unhealthy eating behaviors (e.g., drinking beverage, snacking, and take-away meal habits), and living settings were potentially important factors. From the above, healthy lifestyle and dietary intervention are crucial to weight loss and to keep adolescents healthy.

4.6. Limitations. Several potential limitations of our research should be acknowledged. First, participants were only from a single city of China, and it therefore might be difficult to generalize these findings for adolescents of other regions. Second, alcohol consumption and hepatitis B virus (HBV) and hepatitis $\mathrm{C}$ virus (HCV) infections were less common and out of our considerations $[44,45]$. Third, the lack of comprehensive evaluation of individualized specific characteristics was not enough to conduct a further interventional study on the basis of this study.

\section{Conclusions}

The prevalence of elevated ALT levels differed in gender, age, and criteria among adolescents of Shenzhen. As the high growth period, adolescents have become a focus of public and national health; strengthening healthy education and lifestyle management may be benefits to their healthy growth.
With timely attention to age and gender differences of adolescents during their earlier identification of elevated ALT, more adolescents may avoid the diagnosis of elevated ALTrelated diseases, especially for boys and high school students. Consequently, we should take the prevalence as a predictor and continue to play a warning and preventive role in preparation for further intervention.

\section{Data Availability}

Readers can get the datasets of the current study by contacting the author of Jing Zhang via email (18002534959@163.com) for a reasonable request.

\section{Conflicts of Interest}

The authors declare that they have no competing interests.

\section{Authors' Contributions}

J.Z. and Z.D. conceived and designed the experiments. J.Z., Z.Y.W., and J.P.Z. collected and cleaned the data. J.Z. drafted the manuscript. Z.D. and H.Z. revised the manuscript and interpreted the results. All authors read and approved the final manuscript.

\section{Acknowledgments}

The study was supported by the Baoan District Medical and Health Basic Research Project (grant number: 2018JD088).

\section{Supplementary Materials}

Table S1: number of students with elevated alanine aminotransferase (ALT) stratified by gender and age among the adolescents of Shenzhen, southern China, 2017-2018, separately based on the diagnostic criteria I and II. (Supplementary Materials)

\section{References}

[1] Z. Liu, S. Que, J. Xu, and T. Peng, "Alanine aminotransferaseold biomarker and new concept: a review," International Journal of Medical Sciences, vol. 11, no. 9, pp. 925-935, 2014.

[2] W. R. Kim, S. L. Flamm, A. M. di Bisceglie, H. C. Bodenheimer, and Public Policy Committee of the American Association for the Study of Liver Disease, "Serum activity of alanine aminotransferase (ALT) as an indicator of health and disease," Нераtology, vol. 47, no. 4, pp. 1363-1370, 2008.

[3] P. H. Harada, N. R. Cook, D. E. Cohen, N. P. Paynter, L. Rose, and P. M. Ridker, "Relation of alanine aminotransferase levels to cardiovascular events and statin efficacy," The American Journal of Cardiology, vol. 118, no. 1, pp. 49-55, 2016.

[4] T. Umehara, "Nonalcoholic fatty liver disease with elevated alanine aminotransferase levels is negatively associated with bone mineral density: cross-sectional study in U.S. adults," PLoS One, vol. 13, no. 6, p. e0197900, 2018.

[5] S. Jin, S. Jiang, and A. Hu, "Association between obstructive sleep apnea and non-alcoholic fatty liver disease: a systematic review and meta-analysis," Sleep \& Breathing, vol. 22, no. 3, pp. 841-851, 2018. 
[6] R. W. Smith, C. Korenblum, K. Thacker, H. J. Bonifacio, T. Gonska, and D. K. Katzman, "Severely elevated transaminases in an adolescent male with anorexia nervosa," The International Journal of Eating Disorders, vol. 46, no. 7, pp. 751754, 2013.

[7] S. Zelber-Sagi, S. Toker, G. Armon et al., "Elevated alanine aminotransferase independently predicts new onset of depression in employees undergoing health screening examinations," Psychological Medicine, vol. 43, no. 12, pp. 2603-2613, 2013.

[8] S. E. Mahady and J. George, "Predicting the future burden of NAFLD and NASH," Journal of Hepatology, vol. 69, no. 4, pp. 774-775, 2018.

[9] D. S. Pratt and M. M. Kaplan, "Evaluation of abnormal liverenzyme results in asymptomatic patients," The New England Journal of Medicine, vol. 342, no. 17, pp. 1266-1271, 2000.

[10] X. Y. Wu, C. L. Hu, Y. H. Wan et al., "Higher waist-to-height ratio and waist circumference are predictive of metabolic syndrome and elevated serum alanine aminotransferase in adolescents and young adults in mainland China," Public Health, vol. 126, no. 2, pp. 135-142, 2012.

[11] J. E. Yun, S. Y. Kim, H. C. Kang, S. J. Lee, H. Kimm, and S. H. Jee, "Alanine aminotransferase is associated with metabolic syndrome independently of insulin resistance," Circulation Journal, vol. 75, no. 4, pp. 964-969, 2011.

[12] H. S. Park, J. H. Han, K. M. Choi, and S. M. Kim, "Relation between elevated serum alanine aminotransferase and metabolic syndrome in Korean adolescents," The American Journal of Clinical Nutrition, vol. 82, no. 5, pp. 1046-1051, 2005.

[13] C. E. Ruhl and J. E. Everhart, "Determinants of the association of overweight with elevated serum alanine aminotransferase activity in the United States," Gastroenterology, vol. 124, no. 1, pp. 71-91, 2003.

[14] J. A. Welsh, S. Karpen, and M. B. Vos, "Increasing prevalence of nonalcoholic fatty liver disease among United States adolescents, 1988-1994 to 2007-2010," The Journal of Pediatrics, vol. 162, no. 3, pp. 496-500.e1, 2013.

[15] E. Elinav, I. Z. Ben-Dov, E. Ackerman et al., "Correlation between serum alanine aminotransferase activity and age: an inverted U curve pattern," The American Journal of Gastroenterology, vol. 100, no. 10, pp. 2201-2204, 2005.

[16] M. H. Dong, R. Bettencourt, E. Barrett-Connor, and R. Loomba, "Alanine aminotransferase decreases with age: the Rancho Bernardo Study," PLoS One, vol. 5, no. 12, article e14254, 2010.

[17] C. R. Fermin, A. M. Lee, S. L. Filipp, M. J. Gurka, and M. DeBoer, "Serum alanine aminotransferase trends and their relationship with obesity and metabolic syndrome in United States adolescents, 1999-2014," Metabolic Syndrome and Related Disorders, vol. 15, no. 6, pp. 276-282, 2017.

[18] C. E. Ruhl and J. E. Everhart, "Diurnal variation in serum alanine aminotransferase activity in the US population," Journal of Clinical Gastroenterology, vol. 47, no. 2, pp. 165-173, 2013.

[19] A. Fraser, M. P. Longnecker, and D. A. Lawlor, "Prevalence of elevated alanine aminotransferase among US adolescents and associated factors: NHANES 1999-2004," Gastroenterology, vol. 133, no. 6, pp. 1814-1820, 2007.

[20] R. S. Strauss, S. E. Barlow, and W. H. Dietz, "Prevalence of abnormal serum aminotransferase values in overweight and obese adolescents," The Journal of Pediatrics, vol. 136, no. 6, pp. 727-733, 2000.
[21] J. W. Kim, K. J. Lee, H. R. Yang et al., "Prevalence and risk factors of elevated alanine aminotransferase among Korean adolescents: 2001-2014," BMC Public Health, vol. 18, no. 1, p. 617, 2018.

[22] G. Ramirez-Lopez, S. Morán-Villota, F. Mendoza-Carrera et al., "Metabolic and genetic markers' associations with elevated levels of alanine aminotransferase in adolescents," Journal of Pediatric Endocrinology \& Metabolism, vol. 31, no. 4, pp. 407-414, 2018.

[23] M. Purcell, Y. N. Flores, Z. F. Zhang, E. Denova-Gutiérrez, and J. Salmeron, "Prevalence and predictors of alanine aminotransferase elevation among normal weight, overweight and obese youth in Mexico," Journal of Digestive Diseases, vol. 14, no. 19, pp. 491-499, 2013.

[24] M. van Vliet, I. von Rosenstiel, R. K. Schindhelm, D. P. Brandjes, J. H. Beijnen, and M. Diamant, "The association of elevated alanine aminotransferase and the metabolic syndrome in an overweight and obese pediatric population of multi-ethnic origin," European Journal of Pediatrics, vol. 168, no. 5, pp. 585-591, 2009.

[25] S. G. Samani, R. Kelishadi, A. Adibi, H. Noori, and M. Moeini, "Association of serum alanine aminotransferase levels with cardiometabolic risk factors in normal-weight and overweight children," Iranian Journal of Pediatrics, vol. 21, no. 3, pp. $287-$ 293, 2011.

[26] C. H. Chen, M. H. Huang, J. C. Yang et al., "Prevalence and etiology of elevated serum alanine aminotransferase level in an adult population in Taiwan," Journal of Gastroenterology and Hepatology, vol. 22, no. 9, pp. 1482-1489, 2007.

[27] Z. Ding, J. Zhang, C. Y. Deng, Y. B. You, and H. Zhou, “Association of body mass index with serum alanine aminotransferase in Chinese adolescents: a school-based cross-sectional study," Gastroenterology Report, 2019.

[28] H. K. Park, J. S. Hwang, J. S. Moon, J. A. Lee, D. H. Kim, and J. S. Lim, "Healthy range of serum alanine aminotransferase and its predictive power for cardiovascular risk in children and adolescents," Journal of Pediatric Gastroenterology and Nutrition, vol. 56, no. 6, pp. 686-691, 2013.

[29] M. H. Dong, R. Bettencourt, D. A. Brenner, E. Barrett-Connor, and R. Loomba, "Serum levels of alanine aminotransferase decrease with age in longitudinal analysis," Clinical Gastroenterology and Hepatology, vol. 10, no. 3, pp. 285-290.e1, 2012.

[30] D. G. Le Couteur, F. M. Blyth, H. M. Creasey et al., "The association of alanine transaminase with aging, frailty, and mortality," The Journals of Gerontology. Series A, Biological Sciences and Medical Sciences, vol. 65A, no. 7, pp. 712-717, 2010.

[31] L. Pacifico, F. Ferraro, E. Bonci, C. Anania, S. Romaggioli, and C. Chiesa, "Upper limit of normal for alanine aminotransferase: Quo vadis?," Clinica Chimica Acta, vol. 422, pp. 29-39, 2013.

[32] L. Elizondo-Montemayor, P. A. Ugalde-Casas, L. Lam-Franco et al., "Association of ALT and the metabolic syndrome among Mexican children," Obesity Research \& Clinical Practice, vol. 8, no. 1, pp. e79-e87, 2014.

[33] S. H. Park, N. Y. Heo, J. H. Park et al., "Obesity, insulin resistance, and the risk of an elevated alanine aminotransferase activity in the Korean adolescent population," Journal of Pediatric Endocrinology \& Metabolism, vol. 25, no. 9-10, pp. 945945, 2012.

[34] H. Poustchi, J. George, S. Esmaili et al., "Gender differences in healthy ranges for serum alanine aminotransferase levels in adolescence," PLoS One, vol. 6, no. 6, article e21178, 2011. 
[35] A. P. Kong, K. C. Choi, C. S. Cockram et al., "Independent associations of alanine aminotransferase (ALT) levels with cardiovascular risk factor clustering in Chinese adolescents," Journal of Hepatology, vol. 49, no. 1, pp. 115-122, 2008.

[36] M. E. Mendelsohn and R. H. Karas, "The protective effects of estrogen on the cardiovascular system," The New England Journal of Medicine, vol. 340, no. 23, pp. 1801-1811, 1999.

[37] Y. A. K. Enuameh, G. Adjei, E. Mahama, T. Gyan, and E. Koku, "Effectiveness of population based risk reduction programs for risky sexual behavior among young people in lowand middle-income countries: a systematic review protocol," JBI Database of Systematic Reviews and Implementation Reports, vol. 15, no. 9, pp. 2242-2248, 2017.

[38] T. Westergren, S. Berntsen, M. S. Ludvigsen et al., "Relationship between physical activity level and psychosocial and socioeconomic factors and issues in children and adolescents with asthma: a scoping review protocol," JBI Database of Systematic Reviews and Implementation Reports, vol. 15, no. 2, pp. 269-275, 2017.

[39] T. M. Chaplin and A. Aldao, "Gender differences in emotion expression in children: a meta-analytic review," Psychological Bulletin, vol. 139, no. 4, pp. 735-765, 2013.

[40] D. Matthews, L. Bath, W. Högler, A. Mason, A. Smyth, and M. Skae, "Hormone supplementation for pubertal induction in girls," Archives of Disease in Childhood, vol. 102, no. 10, pp. 975-980, 2017.

[41] K. M. Nelson, K. B. Carey, L. A. J. Scott-Sheldon et al., "Gender differences in relations among perceived family characteristics and risky health behaviors in urban adolescents," Annals of Behavioral Medicine, vol. 51, no. 3, pp. 416-422, 2017.

[42] M. D. Deboer, R. C. Wiener, B. H. Barnes, and M. J. Gurka, "Ethnic differences in the link between insulin resistance and elevated ALT,” Pediatrics, vol. 132, no. 3, pp. e718-e726, 2013.

[43] Y. Kang, S. Park, S. Kim, and H. Koh, "Normal serum alanine aminotransferase and non-alcoholic fatty liver disease among Korean adolescents: a cross-sectional study using data from KNHANES 2010-2015," BMC Pediatrics, vol. 18, no. 1, p. 215, 2018.

[44] Y. H. Ju, H. R. Shin, J. K. Oh et al., "A seroepidemiological study of hepatitis $\mathrm{B}$ and $\mathrm{C}$ virus (HBV and $\mathrm{HCV}$ ) infections in the young population in parts of Busan, Korea," Journal of preventive medicine and public health = Yebang Uihakhoe chi, vol. 37, no. 3, pp. 253-259, 2004.

[45] H. Li, Y. Chen, X. Tian et al., "Comparison of clinical characteristics between lean and obese nonalcoholic fatty liver disease in the northeast Chinese population," Archives of Medical Science - Atherosclerotic Diseases, vol. 4, pp. e191e195, 2019. 